\title{
EDX/XRD-based identification of micrometer-sized domains in scanning electron micrographs of fired clay
}

\author{
Hawraa Kariem • Thomas Kiefer (1D C Christian Hellmich • Wolfgang Gaggl • \\ Andreas Steiger-Thirsfeld $\cdot$ Josef Füssl
}

Received: 17 December 2019/Accepted: 7 July 2020/Published online: 4 August 2020

(C) The Author(s) 2020

\begin{abstract}
The thermal and mechanical properties of bricks are strongly dependent on both the chemical composition and the microstructural features of the used fired clay material. Focussing on the latter, we here identify, in terms of volume fraction, shape, and orientation characteristics, one-to-several micrometer-sized subdomains ("material phases") within the SEM-imaged microstructure of two raw clays fired at
\end{abstract}

Electronic supplementary material The online version of this article (https://doi.org/10.1617/s11527-020-01531-7) contains supplementary material, which is available to authorized users.

H. Kariem · T. Kiefer $(\bowtie) \cdot$ C. Hellmich · J. Füssl Institute for Mechanics of Materials and Structures, TU Wien, Karlsplatz 13/202, 1040 Vienna, Austria e-mail: thomas.kiefer@tuwien.ac.at

H. Kariem

e-mail: hawraa.kariem@tuwien.ac.at

C. Hellmich

e-mail: christian.hellmich@tuwien.ac.at

J. Füssl

e-mail: josef.fuessl@tuwien.ac.at

W. Gaggl

Wienerberger AG, Clay Building Materials Europe, Hauptstrasse 4, 2332 Hennersdorf bei Wien, Austria e-mail: wolfgang.gaggl@wienerberger.com

\section{A. Steiger-Thirsfeld}

University Service Center for TEM (USTEM), TU Wien, Wiedner Hauptstrasse 8-10, 1040 Vienna, Austria e-mail: andreas.steiger-thirsfeld@tuwien.ac.at
880 and 1100 centigrades: (1) quartz grains, (2) muscovite, (3) Fe-Mg mica, (4) feldspar grains, (5) decarbonated dolomite, (6) pores, or (7) binding matrix. This identification rests on the simultaneous use of Scanning Electron Microscopy (SEM) and Energy-dispersive X-ray spectroscopy (EDX), with correspondingly obtained data entering statistical analyses based on the Otsu algorithm, and complemented by minimum grain size and grain shape requirements, as well as by logical exclusion criteria. Crystalline and amorphous phase shares were additionally confirmed by X-ray powder diffraction measurements (PXRD). As for the investigated clays, an increased firing temperature results in dehydroxylation of muscovite, and in a reduced appearance of feldspar grains.

Keywords Fired clay · Microstructure - Quantitative mineralogical analysis $\cdot$ PXRD $\cdot$ SEM-EDX

\section{Introduction}

Clays and clay minerals may provide a wide range of chemical and physical properties [1-3], which renders them suitable for many different applications. Of particular interest in civil engineering and architecture are the thermal conductivity and the mechanical properties of bricks and clay blocks, as they define their sustainability and competitiveness, in 
comparison to other construction materials [4]. During production, the composition of the raw clay and the firing temperature govern the resulting mineralogy and microstructure, which in turn, dictate the thermal and mechanical properties of the fired ceramic body [5-7].

This has motivated the characterization of the chemical and mineralogical composition of fired clay by means of X-ray fluorescence spectrometry and $\mathrm{X}$-ray diffractometry [8]. In addition, the elemental mappings gained by SEM-EDX measurements allow for a distinction between the main phases preliminary observed in the XRD, a method which has been used successfully for concrete, precisely blended-cement pastes [9-12]. A particularly comprehensive multitechnique experimental characterisation of fired clay was provided by Krakowiak et al. [13], who presented XRD spectra, typical EDX composite maps, scanning electron micrographs, mercury intrusion-based pore size distributions, and massive grid nanoindentation data for two types of solid brick. Based on these measurements, Krakowiak et al. [13] propose a hierarchical scheme for the multi-level and multi-component morphology of fired clay. In this context, a material volume of some hundred micrometers characteristic length (called "primary brick") comprises a "glassy" matrix with "silt inclusions" and "micropores". This observation scale is the very focus of the present paper, where we aim at a both more detailed and more quantitative description of the aforementioned "primary brick". More precisely, we introduce and identify one-to-several micrometer-sized (pseudohomogeneous) subdomains within a piece of fired clay, as seen in scanning electron microscopy. Such subdomains are called "material phases" in the framework of continuum micromechanics or random homogenization theory [14-16]. These material phases govern, through their intrinsic "universal" properties as well as through their shapes and volume fractions, the thermal and/or mechanical properties of the aforementioned piece of material. This material phase concept has been very successful in deciphering (micro-)structure - (mechanical) property relations in other civil and bioengineering materials, such as concrete [17, 18], wood [19, 20], or bone [21-23].

Accordingly, we are particularly interested in the volume fractions of these subdomains within a piece of material, and in their geometrical description in terms of aspect ratios and spatial orientations of spheroids approximating the "material phases". Therefore, we here present a novel analysis method combining SEM, EDX, and PXRD data.

The remainder of the paper is organized as follows: after introducing the basic concept in Sect. 2.1, the mineralogical composition of the two raw clays is described in Sect. 2.2. This is followed by a detailed information on clay sample preparation for the SEMEDX analysis (Sect. 2.3). A short discussion on the analysis of the mineralogy of the fired clay samples determined by PXRD measurements is given in Sect. 2.4, providing, in combination with XRF data and the results from the SEM-EDX image analysis, a detailed insight in the chemical composition of the clay matrix. Details on the SEM-EDX analysis are presented in Sect. 2.5, followed by a description of the procedure for a statistical evaluation of the SEM-EDX data, as outlined in Sect. 2.6. The evaluation of threedimensional morphological properties from two-dimensional geometrical data is comprised in Sect. 2.7. Finally, the results are summed up in Sect. 3, and discussed thereafter (Sect. 4).

\section{Materials \& methods}

\subsection{Basic concept}

The aim of the present paper is the identification of the morphology and the mutual position of the material phases which govern the macroscopic thermal and mechanical behaviour of the clay, at the scale of an electron micrograph with a resolution of $195 \mathrm{~nm}$, and a characteristic length of several hundred micrometers. The targeted material phases are: quartz grains, feldspar grains, Fe-Mg mica, muscovite, decarbonated dolomite, pores, and a contiguous amorphous matrix with finely dispersed quartz and feldspar particle inclusions. Quantitative access to these phases stems from careful combination of data from backscattered scanning electron microscopy (SEM), energy dispersive X-ray spectroscopy (EDX), and X-ray powder diffraction (PXRD): Namely, backscattered scanning electron micrographs allow for the identification of decarbonated dolomite, pores, and the aforementioned amorphous matrix, as well as of mineral grains made of quartz or feldspar, but not for the discrimination between the two latter minerals. Similarly, SEM images allow for the discrimination of 
the needle-type morphology of $\mathrm{Fe}-\mathrm{Mg}$ mica and muscovite, from that of all other phases, but not for the discrimination between $\mathrm{Fe}-\mathrm{Mg}$ mica and muscovite. However, quartz and feldspar can be discriminated through EDX analyses, as the presence of silicon indicates quartz; the same is true for $\mathrm{Fe}-\mathrm{Mg}$ mica and muscovite, as the former is suggested by the presence of iron. The comparison of the phase volume fractions as obtained from SEM-EDX, with results from X-ray powder diffraction allows for identification of the shares of quartz and feldspar occurring in grain type and in small particle type, respectively, as well as for the determination of the chemical composition of the amorphous clay matrix. In addition to the phase volume fractions, our quantitative analysis also comprises phase shape and orientation. Therefore, all phases except the amorphous matrix are approximated as spheroids with normals which are axisymmetrically distributed around the anisotropy direction of the transversely isotropic fired clay material. These spheroids appear as ellipses in planes through the anisotropy direction, and the shape and orientation of the latter allows us to re-construct phase-specific aspect ratios orientation distributions of the spheroids.

\subsection{Mineralogical analysis of two raw clay types}

Two raw clays of different origin, currently used for brick production, have been investigated in course of the present paper (thereafter named Clay A and Clay B). Clay A stems from a young tectonic burial and sedimentary basin. It has been deposited in Neogene (Pannonian) in a shallow basin in brackish water environment. Due to the low salt content, fossils from shells (Congeria and Limnocardium) and snails (Melanopsis) are abundant in the sediment and sometimes make up fossil benches. The deposit consists of rather homogenous carbonate bearing clays (dolomite, calcite) with high silt content; the excavation depth of clays currently used for brick production is about $25 \mathrm{~m}$. Clay B originates from an allochthonous fluviatile-swamp-pond type from Pliocene-Peistocene-Holocene (fluvio-lacrusine sedimentation). Most of the deposit consists of almost horizontal clay beds with layers of grey-blue silt with pebbles at the basis (subartesian aquifer). The major part of clays is an alteration of clays of varying color, overlaying karstified limestone bedrock. The total thickness is about $11 \mathrm{~m}$.

PXRD measurements on those raw clay samples used in brick production were performed on a Bruker D8 ADVANCE Eco goniometer, using $\mathrm{CuK}_{\alpha}$ as the radiation source, with a voltage of $40 \mathrm{kV}$ and a current of $25 \mathrm{~mA}$. The diffraction patterns were recorded for angles $2 \theta$ ranging from $5^{\circ}$ to $85^{\circ}$, with a step resolution of $0.01^{\circ}$. A second mineralogical analysis was undertaken on the $<2 \mu$-fraction in order to allow for a distinction between different clay minerals. Therefore, the raw material was blended with hydrogen peroxide to remove the organic parts. After dispersing the suspension with an ultrasonic homogenizer, the separation process was conducted by a centrifuge. Various groups of clay minerals were distinguished according to a set of methods following Schultz [24]. Tempering the sample at $550{ }^{\circ} \mathrm{C}$ allowed for a separation of the main peaks of Chlorite and Kaolinite in the diffraction diagram i.e. the $7 \AA$ peak of Chlorite remains, when tempered, while the $7 \AA$ peak of Kaolinite disappears.

\subsection{Fired clay sample preparation}

From each of the two clay types, four bricks with dimensions of $30 \times 15 \times 125 \mathrm{~mm}^{3}$ were extruded. Two samples of each clay type were fired at $880^{\circ} \mathrm{C}$ (typically used in brick production), and the other two at $1100^{\circ} \mathrm{C}$ (upper limit in tile production, extensive sintering process). For the sample preparation a distilled water-cooled low speed saw (Isomet, Buehler, USA) was used to cut out, from the center of each of the four fired clay bricks, cuboids measuring $6 \times 5 \times 15 \mathrm{~mm}^{3}$ with edges being parallel or orthogonal to the extrusion direction. The latter were then embedded in resin (Agar low viscosity resin, Agar Scientific, UK), followed by oven drying at $60^{\circ} \mathrm{C}$ for $48 \mathrm{~h}$. Thereafter, each cuboid was cut into three specimens with dimensions ranging from $0.5 \times 1 \times 4 \mathrm{~mm}^{3}$ to $2 \times 3 \times 4 \mathrm{~mm}^{3}$, yielding a total of 12 samples, which were then glued, by means of a cyan acrylate adhesive, onto glassy object slides, such that the extrusion direction was oriented either perpendicular to the slides plane, or parallel to one of the long edges of the slide, see Fig. 1, where $x$ relates to the extrusion direction, and $x, y$, and $z$ form a right-handed base 


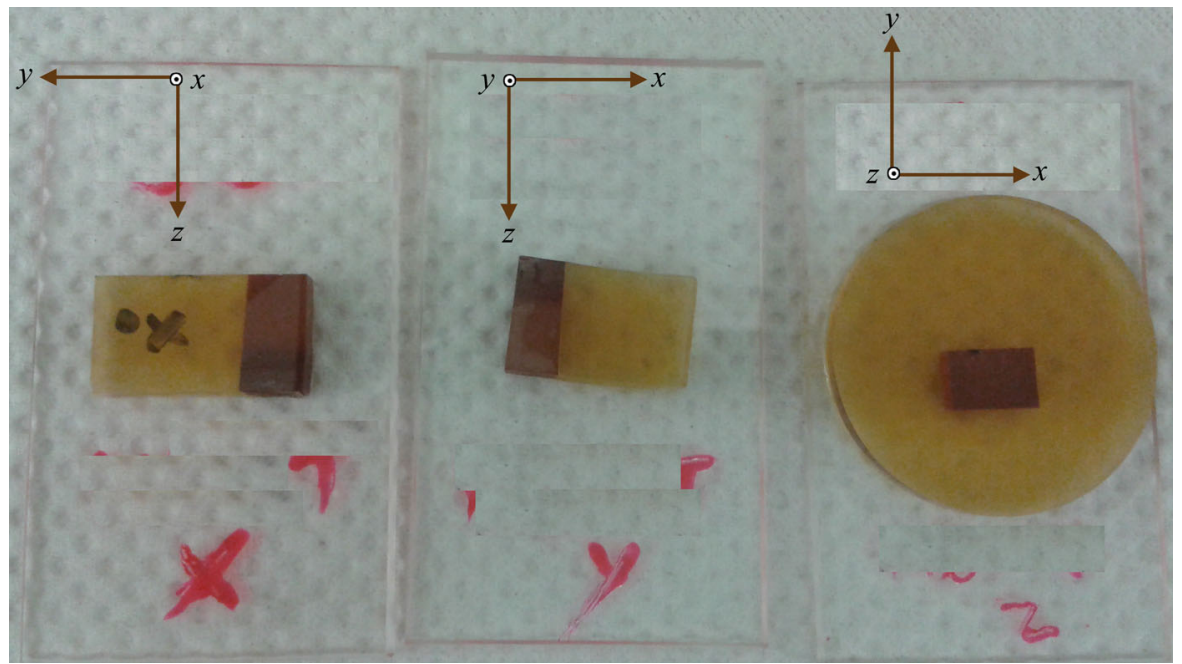

Fig. 1 For each clay and each firing temperatures three specimens were prepared taking the different spatial directions into account; the $x$-axis indicates the extrusion direction, the $z$-axis is the direction of material anisotropy

frame. Each sample is indicated by the type of clay, firing temperature, and the direction which is normal to the image plane. For further sample preparation, the main objective was to obtain a surface of the sample as smooth as possible, which is due to various reasons: obviously, a smooth surface allows for high quality pictures, and ensures that no material phases are quarried out during polishing and get lost during analysis. Furthermore, after scanning the samples with the SEM-EDX, a huge set of measurements will be carried out (and discussed in a subsequent series of papers) on the prepared samples to identify the elastic and thermal properties of the determined material phases by means of nanoindentation [25] and scanning thermal microscopy [26], both methods requiring a very smooth surface to ensure high quality results $[27,28]$. Therefore, the samples underwent an extensive polishing protocol (realised with machine PM5, Logitech, Scotland). First, the specimens were ground by means of silicone carbide papers with grain sizes of 35 microns and 9 microns, respectively. These coarse grinding steps were followed by polishing the specimens with polycrystalline diamonds (DP-Spray P, Struers, Denmark). In all these polishing steps, ethylene glycol was used as a lubricant. After each polishing step, the treated surfaces were examined with a light microscope (Zeiss Axio Imager, Carl Zeiss, Germany), in order to (1) monitor the progressing polishing success in terms of a succession of line- type traces which eventually vanish, and to (2) check the presence of abrasive grains of continuously decreasing size. In order to clean the fired clay specimens from the latter abrasives, they were first rinsed with a mild detergent dissolved in tap water, then rinsed with ethanol [29], then manually polished with a napped cloth (Microcloth, Buehler, USA), and finally rinsed with ethanol. Also the polishing machine was thoroughly cleaned after each polishing step. The fixed parts of the machine were cleaned with paper cloths saturated with distilled water and ethanol, while the removable parts underwent an ultrasonic bath in distilled water. This protocol led to the realization of the smoothest possible surface, characterized by a roughness of only $5 \mathrm{~nm}$, as measured by scanning probe microscopy (TI900 Triboindenter, Hysitron, USA), allowing for high quality pictures and further experiments to derive the mechanical and thermal properties of the material phases (Table 1).

Table 1 Polishing protocol

\begin{tabular}{llll}
\hline Step & Grain size $(\mu \mathrm{m})$ & Time $(\mathrm{min})$ & Plate speed $(\mathrm{rpm})$ \\
\hline 1 & 35 & $3-5$ & 18 \\
2 & 9 & $10-20$ & 18 \\
3 & 3 & $15-23$ & 35 \\
4 & 1 & $15-23$ & 35 \\
5 & 0.25 & $15-23$ & 35 \\
\hline
\end{tabular}




\subsection{Mineralogical analysis of fired samples}

The four fired samples (namely two of each clay type fired at two different temperatures) were analysed by PXRD (X'Pert, Philips, The Netherlands, $\mathrm{CuK}_{\alpha}$ radiation source, angles $2 \theta$ ranging from $5^{\circ}$ to $120^{\circ}$, step size of $0.02^{\circ}$ ). The diffraction pattern entered a quantitative phase analysis (QPA) provided by the software TOPAS (Bruker, USA), being based on the Rietveld method, with the amorphous phase (see Table 4) becoming accessible through the use of Korund (A16SG $1200^{\circ} \mathrm{C} / 48 \mathrm{~h}$, ALCOA, USA) as an internal standard [30,31]. Such is necessary to denote the observed structures from the SEM-EDX pictures to the distinct mineral phases. Additionally, a complete identification of the material phases, including the mineralogy of the glassy matrix phase, can only be accomplished with the information of the PXRD analysis, as the SEM-EDX cannot resolve structures below a certain threshold. The fine mineral particles below this threshold are, as indicated in Sect. 2.6, accounted to the matrix phase, which consists also of an amorphous glassy fraction and mineral structures decomposed during burning. The PXRD is therefore necessary as an advanced information preliminary to the SEM-EDX elemental mapping.

In order to allow for directly comparing the PXRD results with those obtained from SEM-EDX (described in Sects. 2.5 to 2.6), the XRD-derived mass fractions, $M F$, need to be converted to volume fractions, $v f$, according to the following formula, for component $i$ :

$v f_{i}=M F_{i} \times \rho / \rho_{i}$,

with $\rho$ as the mass density of the investigated clay sample, determined by Archimedes' principle [32]; and $\rho_{i}$ as the real mass density of component $i$.

\subsection{SEM-EDX analysis}

The surfaces of the polished samples were investigated by means of Scanning Electron Microscopy (Quanta 200 FEG, FEI, USA). In this context, backscattered electrons were used, and the following settings were applied: an acceleration voltage of $20 \mathrm{kV}$, a spot size of either 3 or 3.5, and the chamber pressure was set to $0.5 \mathrm{mbar}$. For each specimen, a representative area of $271.56 \times 212.16 \mu \mathrm{m}^{2}$ was chosen, and further examined by Energy-Dispersive X-ray spectroscopy (Pegasus XM4, EDAX, USA) with a dwell time of $1000 \mu$ s and 64 frames. In the course of EDX [33], the investigated samples are bombarded with a focussed beam of electrons. The latter create electron vacancies in the inner orbital shells of atoms. This provokes atom-specific X-rays (also called chemical element-specific "characteristic X-rays"), which are emitted when electrons from the orbits further out, "fall" into the aforementioned vacancies. This, in turns, results in chemical elementspecific photons which are quantified in terms of their energies, i.e. $6.391 \mathrm{keV}$ for $\mathrm{Fe}$ (iron), $1.253 \mathrm{keV}$ for $\mathrm{Mg}$ (magnesium), and $1.740 \mathrm{keV}$ for $\mathrm{Si}$ (silicon), the three chemical elements which are specifically targeted in the present application. The number of such photons is then visualised, pixel by pixel, in terms of an 8 bit brightness value [34], yielding elemental maps associated to the representative areas of the investigated specimens. The count of photons is influenced by bremsstrahlung (continuum background X-rays) [34], spectrometer dead time effects [35], and matrix or interelement effects [36]. The latter appears in mixtures of elements due to the differences in elastic and inelastic scattering processes and in the propagation of X-rays through the sample to reach the detector. In order to account for these various effects, the automated background and matrix correction features of the commercial software Genesis (EDAX, USA) were employed. The identification of material phases, or quasi-homogeneous subdomains in terms of continuum micromechanics to which material properties can be assigned by means of a coupled SEMEDX analysis, is described in the following.

\subsection{Phase identification based on SEM-EDX data}

Based on the mineralogical information from the XRD analysis as well as the aforementioned SEM images and elemental maps, consisting of $512 \times 400$ pixels, seven different material phases were identified, in the following way:

- Quartz grains: The Si elemental map was converted from a brightness value image to a binary image, according to Otsu's method [37]. Accordingly, the brightness value histogram was considered as a bimodal distribution, and the threshold separating pixels of one class (belonging to the 
background, and representing anything but quartz grains) from those of the other class (belonging to the foreground, and representing quartz) was determined such that the inter-class variance becomes a maximum. Thereafter, agglomerations of fewer than ten foreground pixels are considered as single $\mathrm{Si}$ spots within the matrix, and were therefore deleted by means of the particle analyser plug-in of ImageJ [38]. The resulting binary image was finally checked against the corresponding backscattered scanning electron micrograph, in order to identify grains which were "falsely glued" by the Otsu method. Such grains were then separated, in the binary image, from each other. This was done by means of introducing a one pixel interface of "separation material", consisting of the matrix phase, which is described further below. Comparing the volume fractions from the PXRD analysis (Table 4) with results from the SEM-EDX (Fig. 12) indicates that fine dispersed quartz particles exist in the matrix.

- Feldspar grains (anorthite): The orthoclase and the anorthite identified by the PXRD cannot be distinguished from each other within the backscattered scanning electron micrograph. Plus, the feldspar grains and the quartz grains cannot be visually separated. This allows for identifying the feldspar grains as all grain-type objects which were not qualified as quartz grains according to the method described before. Fine anorthite grains mineralizing due to higher burning temperatures are, as some quartz particles, dispersed in the matrix and therefore not captured by the SEMEDX.

- Iron-magnesium mica (Fe-Mg mica): All plateshaped features in the backscattered scanning electron micrographs, which contained iron, as indicated by the corresponding elemental maps, were identified as $\mathrm{Fe}-\mathrm{Mg}$ mica. Results from the PXRD and the SEM-EDX analysis show similar results with respect to the volume fractions.

- Light mica (muscovite): All plate-shaped features in the backscattered scanning electron micrographs, which did not contain iron, as indicated by the corresponding elemental maps, were identified as light mica, namely muscovite [39].

- Decarbonated dolomite: This phase was identified as comprising all non-platy features containing magnesium. As the dolomite starts to decompose between $\sim 700{ }^{\circ} \mathrm{C}$ and $800^{\circ} \mathrm{C}$, the identified grains are already at the lower burning temperature of $880^{\circ} \mathrm{C}$ not anymore identified by the XRD, and the grains themselves are mainly remaining structures of the dissolving process of the dolomite. Therefore, the Mg elemental map was converted from a brightness value image to a binary image, according to Yen's algorithm [40]. Accordingly, a cost function representing the discrepancy between the original map and the binary map, as well as the number of bits required for the binary image, was minimized. Afterwards, $\mathrm{Mg}$ pixel agglomerations of fewer than ten pixels as well as with a circularity below two, were deleted by means of the particle analyser plug-in of ImageJ [38]. Within the remaining structures of the decarbonated dolomite, gehlenite, and, with higher burning temperature, diopside as identified by the PXRD may occur.

- Pores: The backscattered scanning electron micrograph was converted from a brightness value image to a binary image, according to Otsu's method [37]. Accordingly, the brightness value histogram was considered as a bimodal distribution, and the threshold separating pixels of one class (belonging to the background, representing anything but pores) from those of the other class (belonging to the foreground, representing pores) was determined such that the inter-class variance becomes a maximum. However, corresponding foreground objects, appearing as dark objects on the SEM, may also contain parts of the decarbonated dolomite phase, so that all sections of the foreground which overlap with the previously determined carbonated dolomite phase, are subtracted from what is considered as the pore space. The remaining foreground pixels form the pore phase, which thereafter undergoes a visual check, in order to split "artificially glued" pores. As before, those pores are separated by a "splitting material" belonging to the matrix phase with a thickness of one pixel. Such is necessary for a correct identification of the phase morphology. The latter concludes the phase identification description.

- Matrix: Structures that could not be uniquely assigned to one of the aforementioned phases were considered to be part of the fired clay matrix. This matrix phase consists of the amorphous fractions, 
(a)

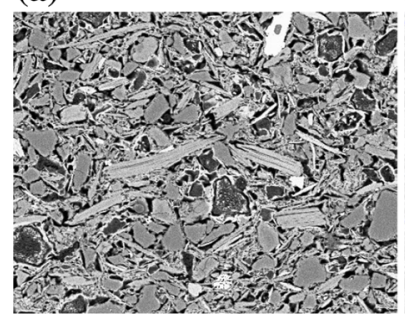

(b)

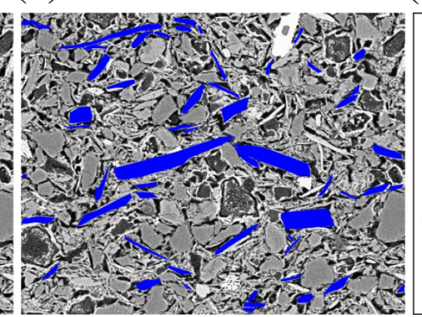

(c)

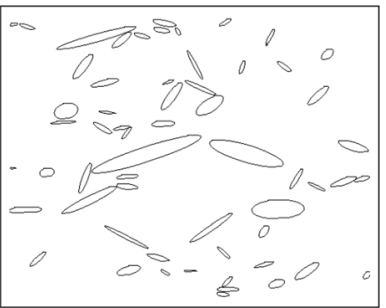

Fig. 2 (a) SEM micrograph of clay A (b) light mica (colored dark blue), as identified from the protocol in Sect. 2.6 (c) best fitting ellipses for the geometrical representation of light mica, yielding aspect ratio, size and orientation for a statistical description of the phase morphology. (Color figure online)

small amounts of minerals, such as haematite and diopside, which cannot be assigned to self-contained structures observable with the SEM-EDX, as well as of fine dispersed mineral particles (like quartz and feldspar). Plus, remaining structures of clay minerals forming new crystal or melting phases, are assigned to the matrix phase. However, all this constituent phases within the glassy matrix phase cannot further be resolved, the microheterogeneous matrix is therefore considered as a quasihomogeneous subdomain in the framework of continuum mechanics.

\subsection{Phase orientation distribution}

In sections through the anisotropy axis of fired clay ( $z-$ axis in Fig. 1), all phases except the matrix phase are approximated as ellipses (see Fig. 2). We interpret the latter as projections of spheroids with phase-specific aspect ratios $\left(a_{n} / R\right), a_{n}$ and $R$ being the spheroids' half-thicknesses and the radii, respectively, and with the orientation distribution of their normal vectors, defined through an angle $\theta$ from the anisotropy direction. In the course of such a projection, the orientation angle $\bar{\theta}$ of the ellipses obtained on the cross sections, being the angle between the anisotropy direction $z$ and the projected normal vector $\overline{\mathbf{n}}$ of the spheroids orientation, is dependent on $\theta$ as well as on $\phi$ (see Fig. 3.)

If transversal isotropy is assumed, then the normal vectors $\mathbf{n}$ of a group of material phases have to be equally distributed along $\phi \in\{0,2 \pi\}$. The projected

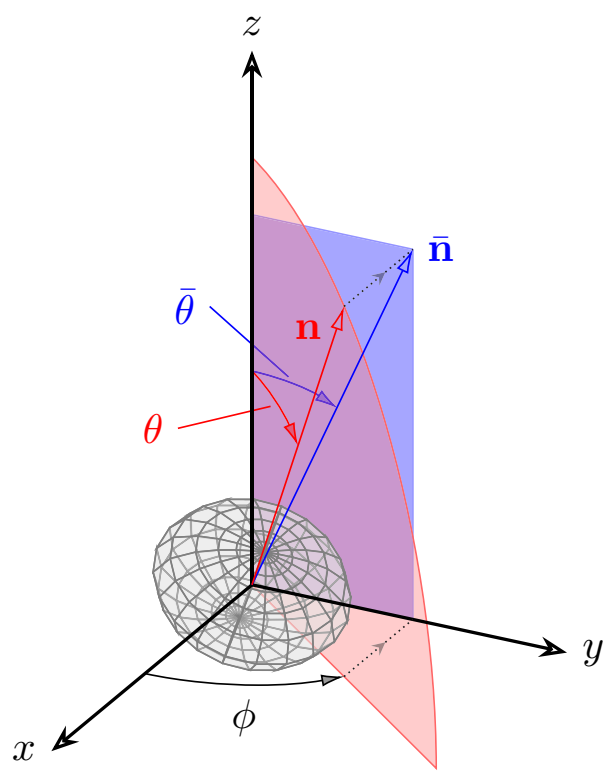

Fig. 3 Projection of the spheroid normal vector $\mathbf{n}(\theta, \phi)$ onto the plane $x=0$

orientation angles, observed on the cross sections (with a plane $x=0$, resp. $y=0$, see Fig. 3), are given to:

$\bar{\theta}=\theta \cdot \sin (\phi)$.

For the case of equally distributed angles $\phi$, the mean value of the orientation distribution by means of the projected orientation angles of a group of material phases can then be calculated to: 
$\bar{\theta}=\frac{\int_{0}^{\pi / 2} \theta \cdot \sin (\phi)}{\pi / 2} d \phi=\frac{\theta}{\pi / 2}$,

thereby giving access to the solid orientation angle $\theta$. When calculating the weighted orientation distribution by means of the standard deviation, defined via:

$\sigma_{\theta}=\sqrt{\frac{\sum_{i=1}^{N} A_{i} / A\left(\theta_{i}-\theta^{*}\right)^{2}}{(N-1) / N}}$,

where $N$ is the number of inclusions of a material phase observed in a cross section, $A_{i} / A$ being the fraction of the inclusion and $\theta^{*}$ the weighted mean value of the orientation angles, then the standard deviation of the projected orientation angles $\bar{\theta}$ correlates linearly with the standard deviation of the solid orientation angles $\theta$, if $\theta^{*}=0$ :

$\sigma_{\theta}=\frac{\pi}{2} \sigma_{\bar{\theta}}$.

To derive the aspect ratio of a spheroid, projected to the plane $x=0$, we consider a spheroid with of the form:

$\frac{x^{2}+y^{2}}{R^{2}}+\frac{z^{2}}{a_{3}^{2}}=1$,

where $x, y, z$ are local coordinates in the direction of local, orthogonal unit vectors $\mathbf{v}_{1}, \mathbf{v}_{2}, \mathbf{n}$ (see Fig. 4). If

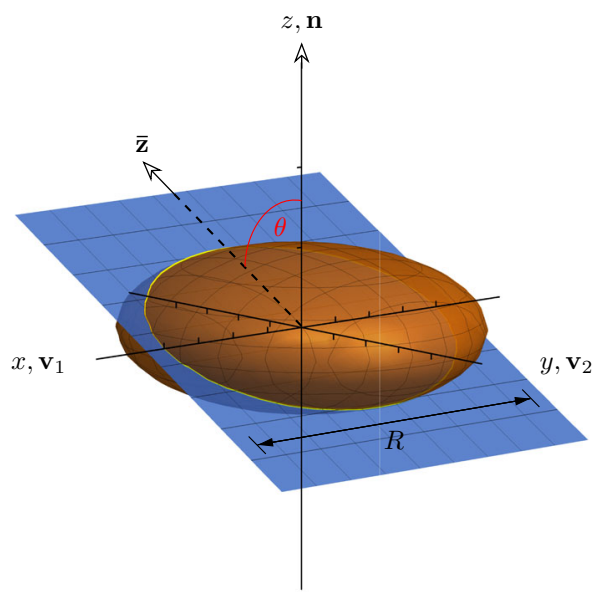

Fig. 4 Cutting a spheroid (brown) with radius $R$ and normal $\overline{\mathbf{n}}$ deviating from the anisotropy direction $\bar{z}$ by angle $\theta$, by means of a plane through the $z$-axis, yields an ellipse (yellow) whose longer half-axis preserves the length of the radius, while its shorter half-axis can be computed from Eq. (11). (Color figure online) we rotate this coordinate system to another system $x, \bar{y}, \bar{z}$ in the plane $x=0$, so that the projection of the spheroid's normal vector $\overline{\mathbf{n}}$ coincides with the $\bar{z}$ direction, then the normal vector of the spheroid in this new system can be written in the form:

$\overline{\mathbf{n}}=\left[\begin{array}{c}\sin (\theta) \\ 0 \\ \cos (\theta)\end{array}\right]$.

The vector $v_{2}$ can be arbitrarily chosen as a unit vector perpendicular to $\overline{\mathbf{n}}$, for example:

$\overline{\mathbf{v}}_{2}=\left[\begin{array}{l}0 \\ 1 \\ 0\end{array}\right]$.

Resulting for the third unit vector to be:

$\overline{\mathbf{v}}_{1}=\overline{\mathbf{n}} \times \overline{\mathbf{v}}_{2}=\left[\begin{array}{c}-\cos (\theta) \\ 0 \\ \sin (\theta)\end{array}\right]$.

Having the local coordinate system of basis vectors we can immediately write for any point $[\bar{x}, \bar{y}, \bar{z}]=\bar{x} \overline{\mathbf{v}}_{1}+\bar{y} \overline{\mathbf{v}}_{2}+\bar{z} \overline{\mathbf{n}}=x \mathbf{v}_{1}+y \mathbf{v}_{2}+z \mathbf{n}, \quad$ from which we derive, using orthonormality:

$$
\begin{aligned}
{\left[\begin{array}{c}
\bar{x} \\
\bar{y} \\
\bar{z}
\end{array}\right] } & =\left[\mathbf{v}_{1} \mathbf{v}_{2} \mathbf{n}\right]\left[\begin{array}{l}
x \\
y \\
z
\end{array}\right] \Longleftrightarrow\left[\begin{array}{c}
x \\
y \\
z
\end{array}\right]=\left[\begin{array}{c}
\overline{\mathbf{v}}_{1}^{\mathrm{T}} \\
\overline{\mathbf{v}}_{2}^{\mathrm{T}} \\
\overline{\mathbf{n}}^{\mathrm{T}}
\end{array}\right]\left[\begin{array}{c}
\bar{x} \\
\bar{y} \\
\bar{z}
\end{array}\right] \\
& =\left[\begin{array}{ccc}
-\cos (\theta) & 0 & \sin (\theta) \\
0 & 1 & 0 \\
\sin (\theta) & 0 & \cos (\theta)
\end{array}\right]\left[\begin{array}{c}
\bar{x} \\
y \\
\bar{z}
\end{array}\right] .
\end{aligned}
$$

Using this transformation rule, we can transform Eq. (6) into the rotated system. Considering the projection into plane $x=0$, we finally get:

$\frac{\bar{y}^{2}}{R^{2}}+\frac{\bar{z}^{2}}{\bar{a}_{3}^{2}}=1, \quad \overline{a_{3}}=\sqrt{R^{2} \cdot \sin ^{2} \theta+a_{3}^{2} \cdot \cos ^{2} \theta}$.

The obtained aspect ratio $a_{1} / \overline{a_{3}}$ has to be identical to those obtained from the 2D images (see Table 7), thereby giving access to the morphology of the spheroids (listed in Table 8). 


\section{Results and discussion}

\subsection{Mineralogical characterization}

\subsubsection{Raw clay samples}

According to the PXRD results on the unfired samples, clay A contained carbonates (calcite and dolomite, see Table 2), while clay B is free of such, containing more quartz. Additionally, both show some shares of phyllosilicates (chlorite, muscovite and kaolinite) as well as some feldspars (albite and microcline), see Table 2.

The PXRD on the $<2 \mu$-fraction reveals that clay A appears to be dominated by smectite and illite, containing also chlorite and kaolinite. In contrast, Clay $\mathrm{B}$ (poor in $\mathrm{Ca}$ ), is dominated by vermiculite, with some constituents of illite, chlorite and kaolinite (see Table 3).

\subsubsection{Fired clay samples}

Results from the PXRD analysis for the fired clay are given in Table 4.

Clay A, representing a clay rich in carbonates, shows, in agreement with literature [42], a wide compositional variability with respect to the carbonate-poor clay B (Table 4, Fig. 12). This can be explained by the breakdown of chlorite [43] at $\sim 500^{\circ} \mathrm{C}$, as well as calcite and dolomite at $\sim 850^{\circ} \mathrm{C}$, releasing $\mathrm{Ca}$ and $\mathrm{Mg}$, which produce a

Table 2 Mineralogical composition in [mass-\%] of the raw mixtures of clay A and clay B, as obtained by PXRD

\begin{tabular}{lll}
\hline Phases & $\begin{array}{l}\text { Clay A } \\
{\left[\%_{\text {wt }}\right]}\end{array}$ & Clay B \\
\hline Quartz & 39 & 60 \\
Chlorite & 7 & 3 \\
Muscovite & 26 & 16 \\
Paragonite & 3 & - \\
Kaolinite & - & 3 \\
Albite & 11 & 11 \\
Microcline & 6 & 8 \\
Calcite & 3 & - \\
Dolomite & 7 & - \\
Sum & 100 & 100 \\
\hline
\end{tabular}

Table 3 Mineralogical composition in [mass-\%] of the raw mixtures of clay A and clay B, as obtained by PXRD (only clay minerals)

\begin{tabular}{lll}
\hline Clay minerals & $\begin{array}{l}\text { Clay A } \\
{\left[\%_{\text {wt }}\right]}\end{array}$ & Clay B \\
\hline Smectite & 58 & - \\
Illite & 23 & 14 \\
Chlorite & 10 & 11 \\
Kaolinite & 8 & 15 \\
Vermiculite & 1 & 60 \\
Sum & 100 & 100 \\
\hline
\end{tabular}

compositional array of new forming minerals with the surrounding clay minerals [44]. With higher burning temperature, clay A shows a significant decrease in the quartz content, something which can be typically expected for carbonate-containing clays [45, 46]. This occurs due to a sudden onset of a melting phase at temperatures of $\sim 1080^{\circ} \mathrm{C}$, which can be observed specifically at the boundaries of the quartz grains (see Fig. 5a). Riccardi et al. [42] reported that such transformation at a wider reaction layer around quartz, beginning at temperatures of $\sim 1050^{\circ} \mathrm{C}$, can be observed in Ca-rich clays only, and can be explained with the nucleation of newly forming anorthite round the quartz grains due to the presence of diffused calcite in the clay matrix. Besides, the high-temperature modification cristobalite is forming at a burning temperature of $1100^{\circ} \mathrm{C}$. The highest amount of newly forming crystals within clay $\mathrm{A}$ is represented by anorthite, which has also been previously reported by other authors [45], followed by small amounts of diopside and the aforementioned cristobalite. As the raw material contains dolomite and calcite, which decarbonate at temperatures of $\sim 700^{\circ} \mathrm{C}$ to $800^{\circ} \mathrm{C}$, these minerals are not detected by the PXRD within our samples. Also, the PXRD results for the fired clay at $880^{\circ} \mathrm{C}$ do not clearly indicate the presence of $\mathrm{Mg}$ and $\mathrm{Ca}$ containing minerals, oxides or hydroxides, a problem which has already been adressed by Cultrone et al. [47]. However, the original microtextural site can still be observed at a burning temperature of $880^{\circ} \mathrm{C}$ in the SEM, significantly enriched in $\mathrm{Mg}$ and $\mathrm{Ca}$ (see Fig. 12a). With higher burning temperatures, and the former dolomite and calcite grains fully reacted, only ring-like textures remain still visible (see Fig. 12), a 
Table 4 Mineralogical composition in [mass-\%] of clay A and B at two different firing temperatures obtained by X-ray diffraction
The volume fractions are calculated using Archimedes principle (see Eq. 1), the densities of the minerals are taken from the RRUFF $^{\mathrm{TM}}$ Project database [41]

\begin{tabular}{|c|c|c|c|c|c|}
\hline & & \multicolumn{2}{|l|}{ Clay A } & \multicolumn{2}{|l|}{ Clay B } \\
\hline & & $880^{\circ} \mathrm{C}$ & $1100{ }^{\circ} \mathrm{C}$ & $880{ }^{\circ} \mathrm{C}$ & $1100^{\circ} \mathrm{C}$ \\
\hline \multirow[t]{2}{*}{ Quartz } & [mass-\%] & $33 \pm 3$ & $19 \pm 2$ & $48 \pm 3$ & $35 \pm 2$ \\
\hline & [vol-\%] & $21.4 \pm 1.9$ & $13.8 \pm 1.4$ & $35.3 \pm 2.2$ & $30.2 \pm 1.7$ \\
\hline \multirow[t]{2}{*}{ Hematite } & [mass-\%] & $2 \pm 1$ & $2 \pm 1$ & $2 \pm 1$ & $4 \pm 1$ \\
\hline & [vol-\%] & $0.6 \pm 0.3$ & $0.7 \pm 0.4$ & $0.7 \pm 0.4$ & $1.7 \pm 0.4$ \\
\hline \multirow[t]{2}{*}{ Muscovite } & [mass-\%] & $6 \pm 2$ & - & $4 \pm 1$ & - \\
\hline & [vol-\%] & $3.6 \pm 1.2$ & - & $2.7 \pm 0.7$ & - \\
\hline \multirow[t]{2}{*}{ Anorthite } & [mass-\%] & $10 \pm 3$ & $21 \pm 4$ & $4 \pm 2$ & - \\
\hline & [vol-\%] & $6.1 \pm 1.8$ & $14.3 \pm 2.7$ & $2.8 \pm 1.4$ & - \\
\hline \multirow[t]{2}{*}{ Orthoclase } & [mass-\%] & $4 \pm 1$ & - & - & - \\
\hline & [vol-\%] & $2.6 \pm 0.7$ & - & - & - \\
\hline \multirow[t]{2}{*}{ Gehlenite } & [mass-\%] & $3 \pm 1$ & - & - & - \\
\hline & [vol-\%] & $1.7 \pm 0.6$ & - & - & - \\
\hline \multirow[t]{2}{*}{ Diopside } & [mass-\%] & - & $3 \pm 1$ & - & - \\
\hline & [vol-\%] & - & $1.7 \pm 0.6$ & - & - \\
\hline \multirow[t]{2}{*}{ Cristobalite } & [mass-\%] & - & $2 \pm 1$ & - & - \\
\hline & [vol-\%] & - & $1.6 \pm 0.8$ & - & - \\
\hline \multirow[t]{2}{*}{ Mullite } & [mass-\%] & - & - & - & $15 \pm 2$ \\
\hline & [vol-\%] & - & - & - & $10.7 \pm 1.4$ \\
\hline amorph & [Mass-\%] & $41 \pm 8$ & $53 \pm 7$ & $42 \pm 7$ & $46 \pm 6$ \\
\hline
\end{tabular}

(a)

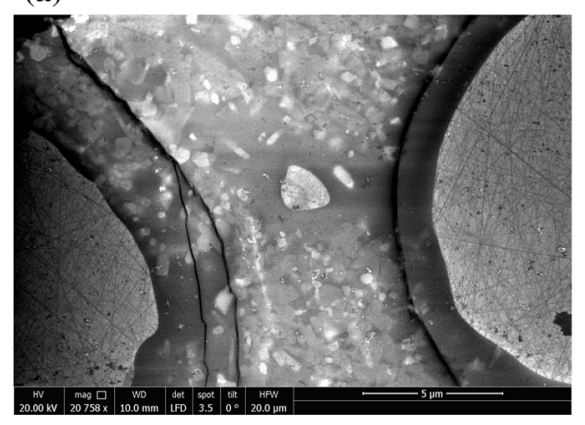

(b)

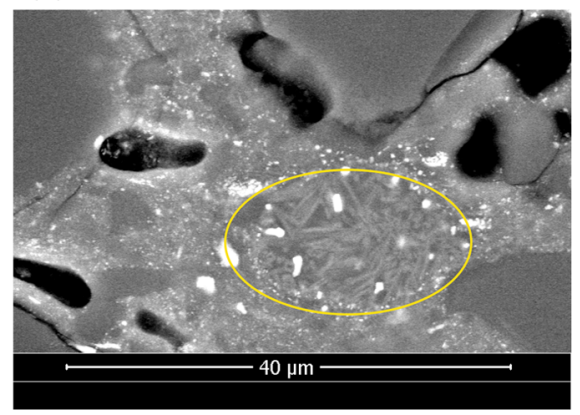

Fig. 5 SEM micrographs of (a) quartz grains melting due to higher burning temperatures at $1100^{\circ} \mathrm{C}$ within clay A and (b) newly formed mullite crystals in the "glassy" vitrified matrix phase of clay B due to higher burning temperatures at $1100^{\circ} \mathrm{C}$

phenomenon which has also been reported by Riccardi et al. [42]. The calcite forms, in addition with siliciumdioxide of clay minerals, anorthite and gehlenite, while gehlenite as an intermediate forming mineral converts with $\mathrm{SiO}_{2}$ from clay minerals or quartz grains to anorthite with its higher silicid acid content during the sinter reaction [45, 48]. Muscovite, paragonite and kaolinite, along with the microcline, are partly dissipated during the firing process, but still observable as muscovite and orthoclase at a burning temperature of $880^{\circ} \mathrm{C}$. The muscovite and orthoclase then dissolve, as expected from literature [47, 49], at high burning temperatures. Regarding the constant amount of hematite, the following can be stated: all calciumaluminum silicates accept varying amounts of iron within their mineral structures $[45,50]$, which is why only little amounts of pure hematite are found, and no new crystallization can be observed at higher temperatures. Finally, the absence of albite at burning temperatures exceeding $850{ }^{\circ} \mathrm{C}$ is caused by its change of the chemical composition by reaction with the surrounding clay matrix [44], when a rim of 
Table 5 Chemical composition in [mass-\%] of clay $\mathrm{A}$ and $\mathrm{B}$ at two different firing temperatures obtained by X-ray fluorescence spectrometry major elements [mass-\%]
$\mathrm{K}$-feldspars is constantly growing, reducing the $\mathrm{Na}$ content in the former albite crystal.

Clay B, which is poor in carbonates, shows a substantial different behaviour. As iron is not trapped within the lattice of calcium-aluminum silicates in clay $\mathrm{B}$, an increase in hematite content can be found with higher burning temperature [43]. As with clay A, quartz and muscovite dissolve (partly) at burning temperatures of $1100{ }^{\circ} \mathrm{C}$, while the only newly formed mineral is mullite at $\sim 1050{ }^{\circ} \mathrm{C}$ [46], observed also in SEM pictures (see Fig. 5b). Cultrone et al. [49] indicate that large formations of mullite out of the melt phase can be observed. Such would explain the decrease of $\mathrm{Al}_{2} \mathrm{O}_{3}$ within the matrix at a burning temperature of $1100{ }^{\circ} \mathrm{C}$, see Table 6. Compared to clay A, a more distinctive melt formation can already be observed at the lower burning temperature of $880^{\circ} \mathrm{C}$. This is caused by the decomposition of carbonates in clay A, promoting the fast crystallization of calcium-aluminum silicates, at the expense of the formation of a melt phase [43].

As expected, the volume fraction of $\mathrm{SiO}_{2}$ within the amorphous part of the matrix is increasing with a higher burning temperature due to the formation of a melting phase (see Fig. 5a) and the decomposition of clay minerals. While the content of $\mathrm{Al}_{2} \mathrm{O}_{3}$ in the matrix of clay A stays constant, the newly forming of mullite crystals in the matrix (see Fig. 5b) reduces the fraction of $\mathrm{Al}_{2} \mathrm{O}_{3}$ in clay $\mathrm{B}$. The small reduction of $\mathrm{MgO}_{2}$ in clay $\mathrm{A}$ can be explained by the newly forming diopside, while the reduction of $\mathrm{Fe}_{2} \mathrm{O}_{3}$ in clay
B can be assigned to the increasing mineral content of hematite.

To derive the chemical composition of the matrix, containing the amorphous fraction, mineral structures decomposed during burning as well as fine dispersed mineral grains, the PXRD data can be used to estimate the chemical composition. Besides the physical properties, the RRUFF ${ }^{\mathrm{TM}}$ Project database [41] contains the chemical composition of minerals, which we can use in combination with the results from the PXRD to compute the mass fractions of molecules recorded by the XRD. Comparison of the results with the XRF data (see Table 5) reveals the chemical composition of the amorphous fraction, given in Table 6.

\subsection{SEM-EDX image analysis}

Otsu's method applied to the Si elemental maps, combined with the requirement of 10 pixel minimum grain size, and after "splitting artificially glued grains" according to an SEM-based visual check, allows for satisfactory identification of the quartz grains in all investigated samples, compare images labelled with (a) and (c), respectively, in Fig. 6 (see also supplementary material). Grain-type objects, not identified as quartz grains by Otsu's method applied on the Si elemental maps, thereafter were assigned as feldspar grains (see Fig. 7), following the protocol in Sect. 2.6. Overlaying plate-shaped features in the backscattered scanning electron micrographs (see Fig. 8a) with the corresponding Fe elemental maps 
Table 6 Chemical composition of the amorphous fraction in [mass-\%] of clay A and B at two different firing temperatures, obtained by subtraction of the chemical composition of the minerals identified by the PXRD

(Table 4) from results of the XRF (Table 5)

(a)

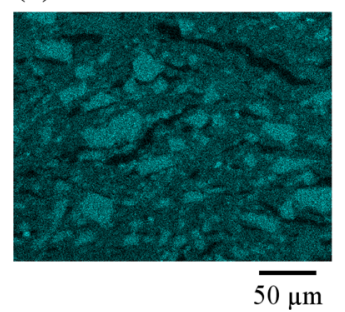

(b)

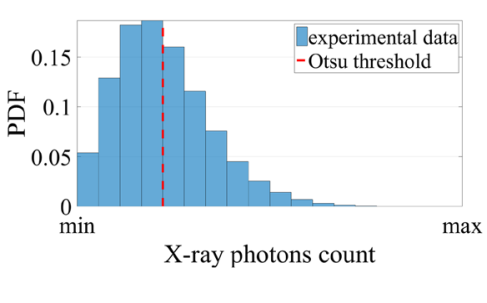

(c)

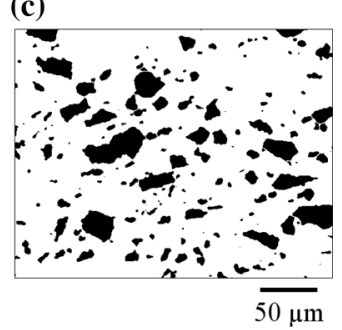

Fig. 6 Quartz grain identification in sample "clay B $1100^{\circ} \mathrm{C} x$ ”, area c: (a) Si elemental map; (b) histogram of Si elemental map, with Otsu threshold; (c) binary image, with quartz grains in black

(a)

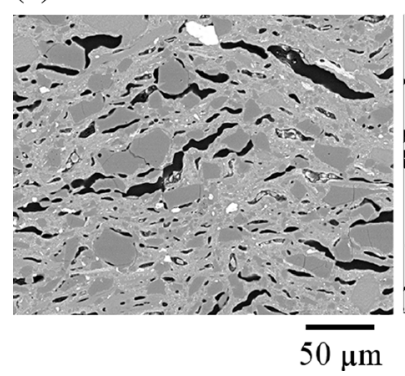

(b)

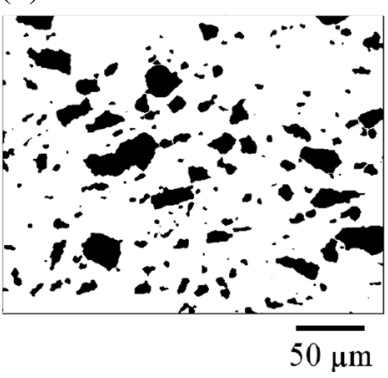

(c)

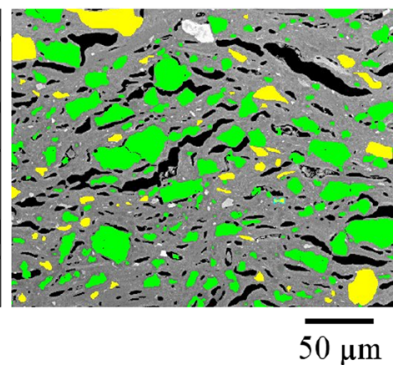

Fig. 7 Feldspar identification in sample "clay B $1100^{\circ} \mathrm{C} x$ ”: (a) backscattered scanning electron micrograph; (b) quartz grains from Fig. 6 (c) backscattered scanning electron micrograph, with feldspar grains in yellow and quartz grains in green. (Color figure online)

(see Fig. 8b) allows for satisfactory identification of $\mathrm{Fe}-\mathrm{Mg}$ mica and of muscovite, respectively, see cyan and blue feature in Fig. 8c. Yen's algorithm applied to the $\mathrm{Mg}$ elemental maps, combined with the requirement of 10 pixels minimum grain size and of 0.2 minimum circularity, allowed for the identification of the decarbonated dolomite phase in all investigated samples, compare images labelled with (a) and (c), respectively, in Fig. 9 (see also supplementary material). Otsu's method applied to the backscattered scanning electron micrographs, and subsequent subtraction of all foreground features which are already assigned to the decarbonated dolomite phase, followed by splitting "artificially glued pores" according to an SEM-based visual check, allows for satisfactory identification of the pore phase, see Fig. 10 (see also 
(a)

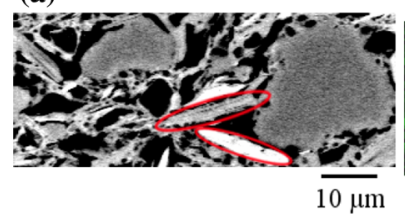

(b)

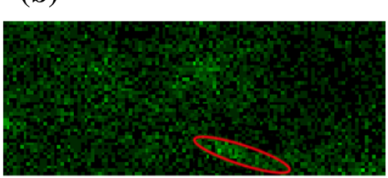

(c)

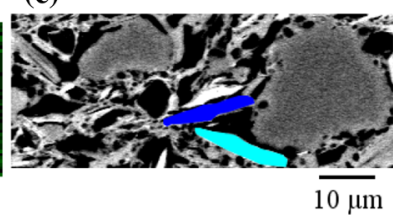

Fig. 8 Identification of Fe-Mg mica of muscovite, in sample "clay A $880^{\circ} \mathrm{C} x$ ": (a) detail of scanning backscattered electron micrograph with two encircled plate-shaped features; (b) detail of corresponding Fe-map with encircled location of higher brightness values; (c) Fe-Mg mica in cyan, muscovite in blue. (Color figure online)

(a)

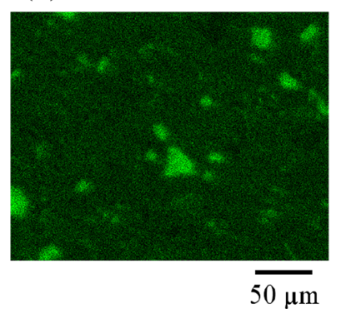

(b)

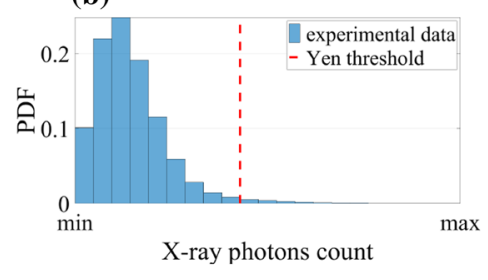

(c)

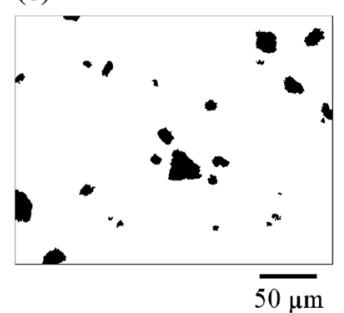

Fig. 9 Decarbonated dolomite identification in sample "clay A $880^{\circ} \mathrm{C} x$ ": (a) elemental map of $\mathrm{Mg}$; (b) histogram of Mg elemental map, with Yen threshold; (c) binary image, with decarbonated dolomite particles in black

(a)

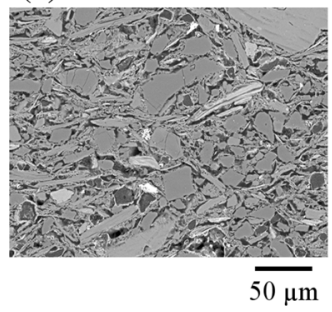

(b)

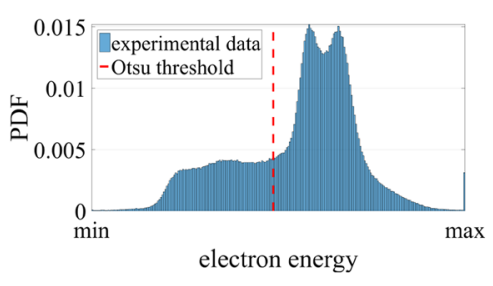

(c)

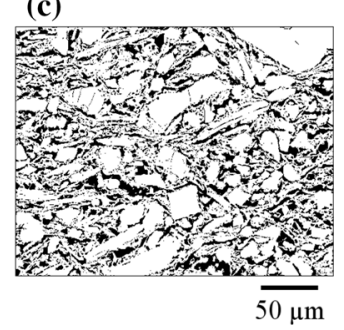

Fig. 10 Pore identification in sample "clay A $880^{\circ} \mathrm{C} y$ ": (a) backscattered electron image; (b) histogram of backscattered electron image, with Otsu threshold; (c) binary image, with pore phase in black
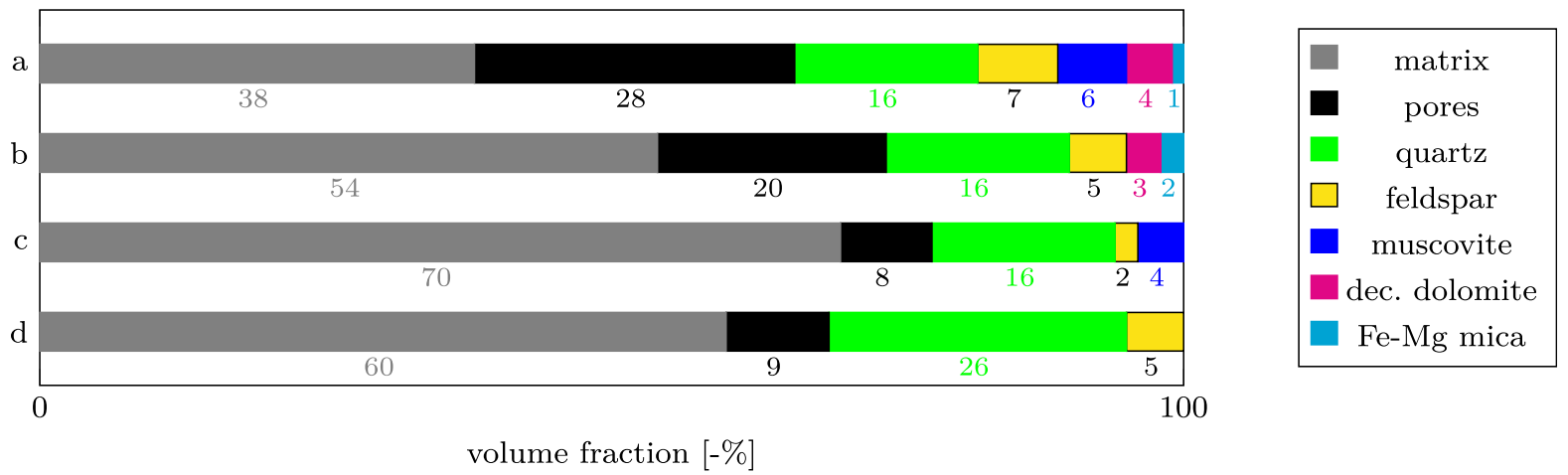

Fig. 11 Sample-specific volume fractions, as obtained from SEM-EDX-based statistical analysis: (a) sample "clay A $880{ }^{\circ} \mathrm{C}$ ", (b) sample "clay B $880^{\circ} \mathrm{C}$ ", (c) sample "clay A $1100^{\circ} \mathrm{C}$ ", (d) sample "clay B $1100^{\circ} \mathrm{C}$ " 
(a)

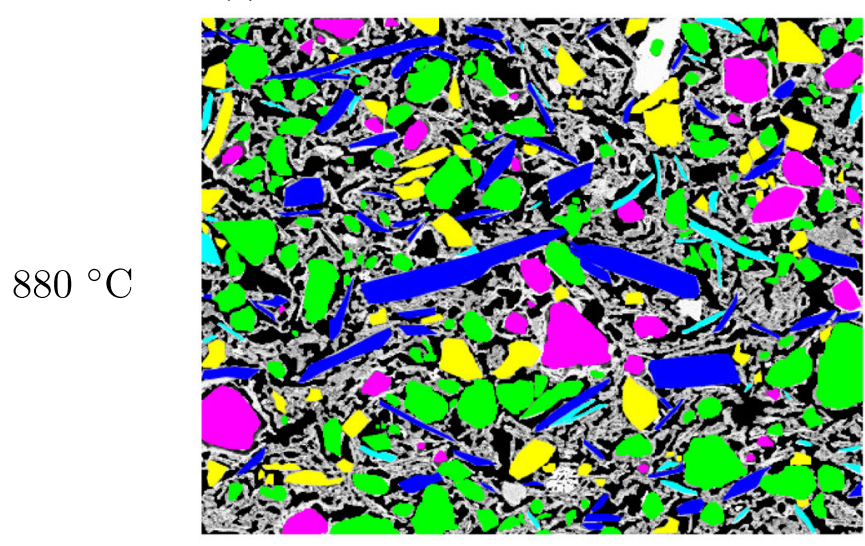

(c)

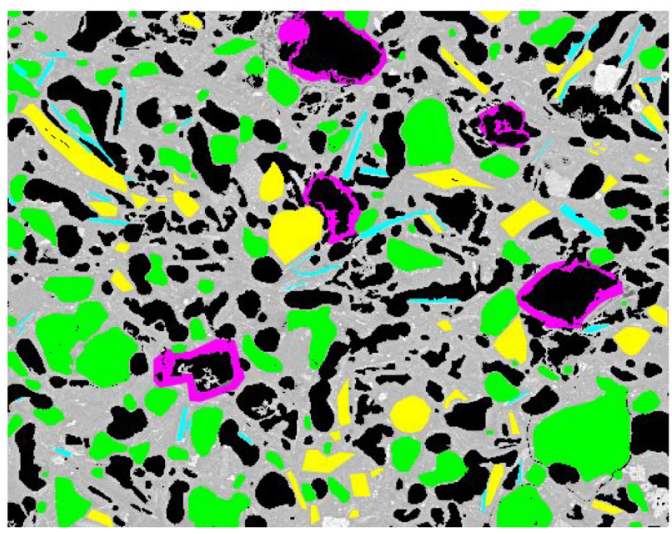

(b) clay B

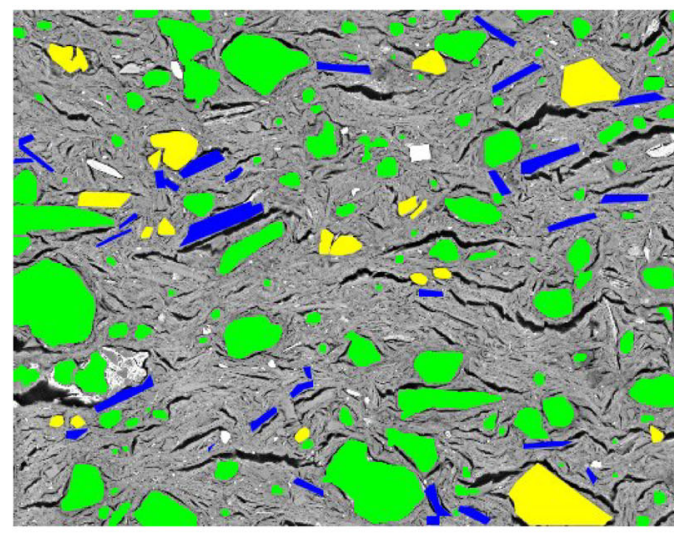

(d)

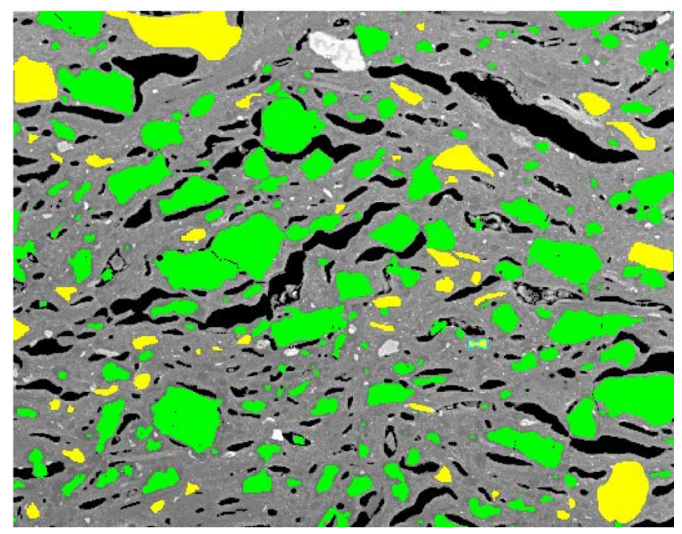

$\square$ quartz grain muscovite $\square$ Fe-Mg mica $\square$ feldspar grain

Fig. 12 Colour-based phase identification, on representative areas shown in Fig. 13: (a) from sample "clay A $880^{\circ} \mathrm{C} x$ ", (b) from sample "clay B $880^{\circ} \mathrm{C} x$ ", (c) from sample "clay A $1100^{\circ} \mathrm{C} x$ ", (d) from sample "clay B $1100^{\circ} \mathrm{C} x$ "; the $x$-axis indicates the extrusion direction. Dimensions $271.56 \times 212.16 \mu \mathrm{m}^{2}$. (Color figure online)

supplementary material). As the aforementioned binary maps are representative for the entire (spatial) clay samples, their mean grey values give direct access to the phase volume fraction, see Fig. 11 . At $880^{\circ} \mathrm{C}$ firing temperature, the quartz grain volume fraction obtained from SEM-EDX are consistently lower than the quartz volume fraction from $\mathrm{XRD}$, compare
Fig. 11a, b and Table 4. This indicates that at this lower firing temperature, quartz appears not only in grain form, but also as fine dispersed particles within the matrix phase. At $1100^{\circ} \mathrm{C}$ firing temperature, the quartz grain volume fractions obtained from SEMEDX agree very well with the XRD-derived quartz volume fraction, compare Fig. 11c, d and Table 4. 
(a) clay A

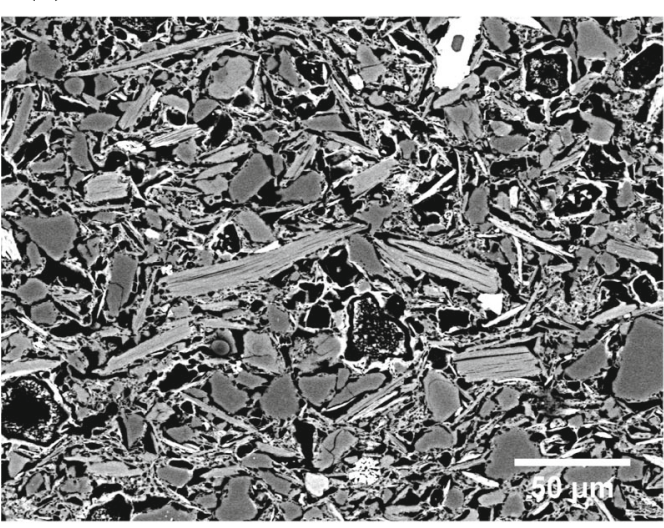

(c)

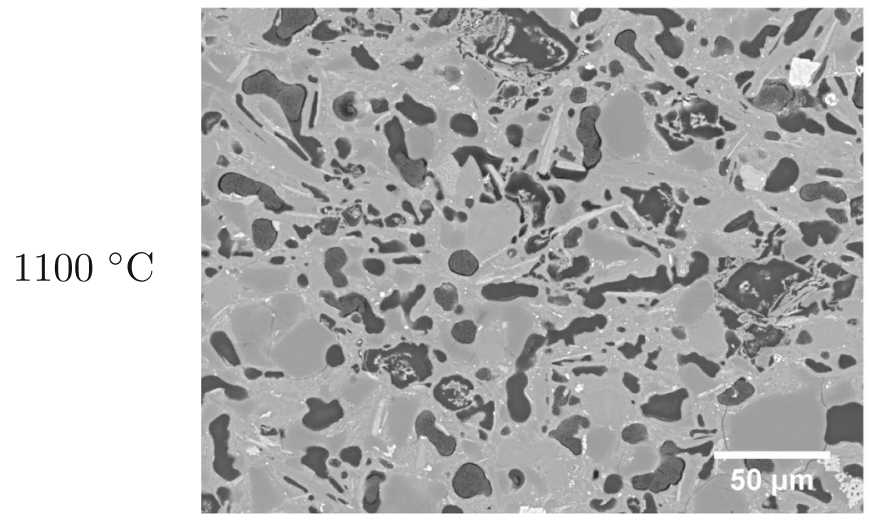

(b)

\section{clay B}

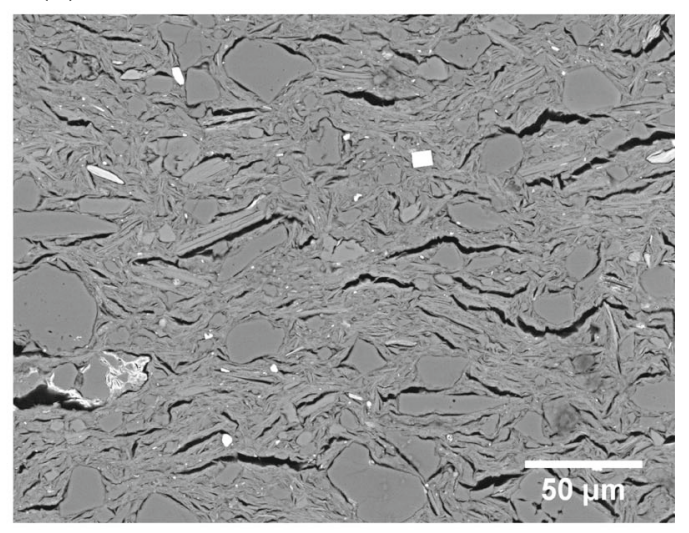

(d)

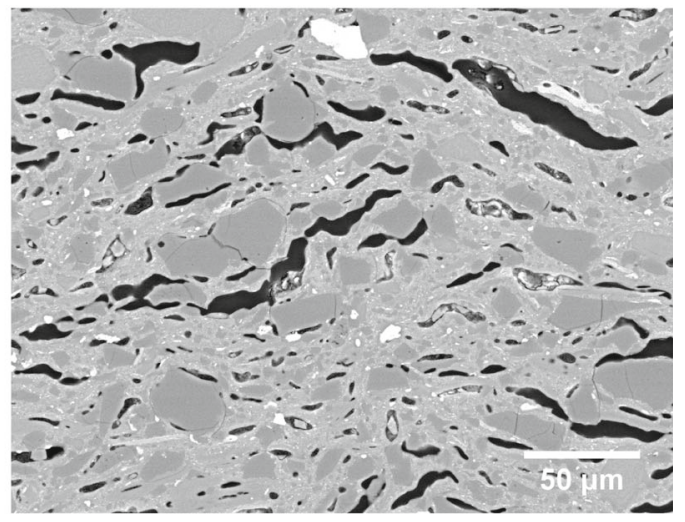

Fig. 13 Selection of backscattered scanning electron micrographs: (a) from sample "clay A $880^{\circ} \mathrm{C} x$ ", (b) from sample "clay B $880^{\circ} \mathrm{C} x$ ", (c) from sample "clay A $1100^{\circ} \mathrm{C} x$ ", (d) from sample "clay B $1100^{\circ} \mathrm{C} x$ "; the $x$-axis indicates the extrusion direction

This indicates that at this higher firing temperature, quartz appears virtually exclusively in the form of several micrometer-sized grains. At $880^{\circ} \mathrm{C}$ firing temperature, the volume fractions of muscovite obtained from SEM-EDX agree fairly well with those derived from XRD, compare Fig. 11a, b and Table 4. At $1100{ }^{\circ} \mathrm{C}$ firing temperature, the SEM-EDX analysis confirms the disappearance of muscovite, as already known from the PXRD results.

The higher porosity of clay A (compare Fig. 11a, c to $11 \mathrm{~b}, \mathrm{~d}$ ) indicates the higher carbonate content in its raw state [49], cf. 2.3. Carbonate-bearing clays are known to be rather heterogeneous [45], which is consistent with clay A exhibiting indeed more material phases than clay B (compare Fig. 11a, c to $11 \mathrm{~b}$, d). $1100^{\circ} \mathrm{C}$ ignition-induced disappearance of pores smaller than $2 \mu \mathrm{m}$ (see Fig. 14) is consistent with the vitrification processes described by Cultrone et al. [49] and Zouaoui et al. [51]. However, micropores of size below the resolution of the SEM-images with $0.192 \mu \mathrm{m}$ pixel size may still prevail. This is suggested by an intravoxel microCT-image analysis which was experimentally validated by weighing tests and mercury intrusion porosimetry [52]. Correspondingly obtained porosity values are about $8 \%$ higher than those obtained from present SEM-EDX results of Fig. 11a, c. These additional pores of clearly submicrometer size are part of the matrix phase. 
(a)

clay A

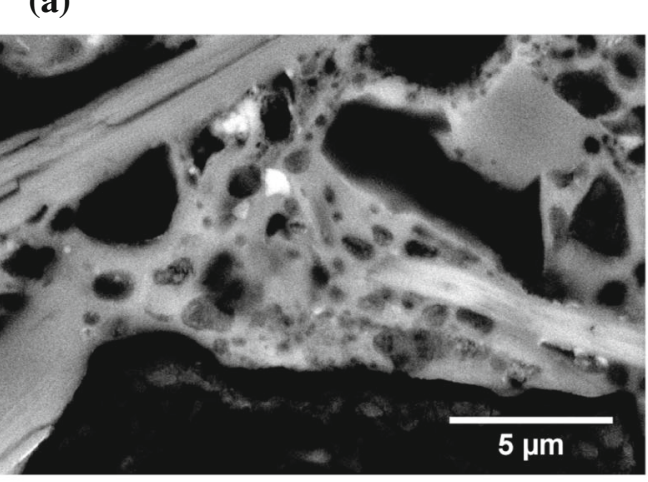

(c)

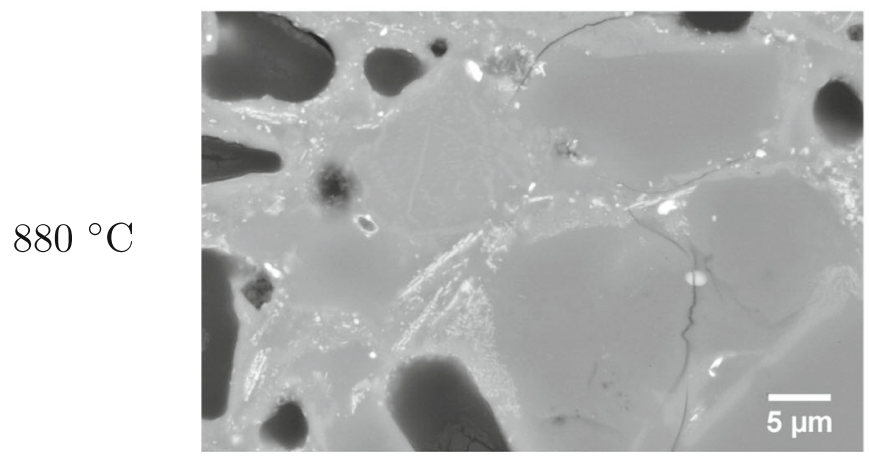

(b)

clay B

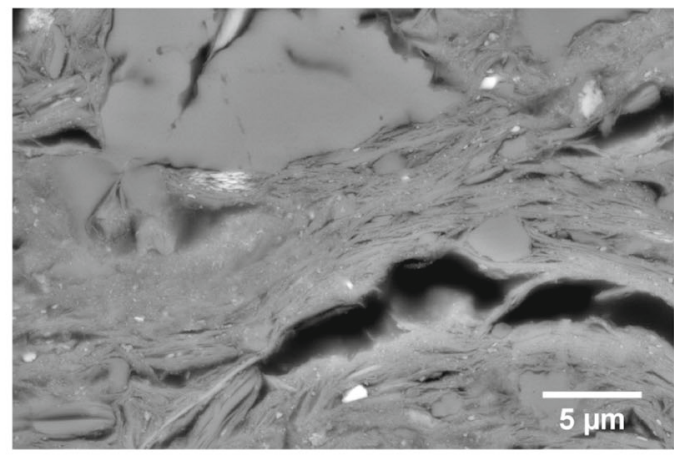

(d)

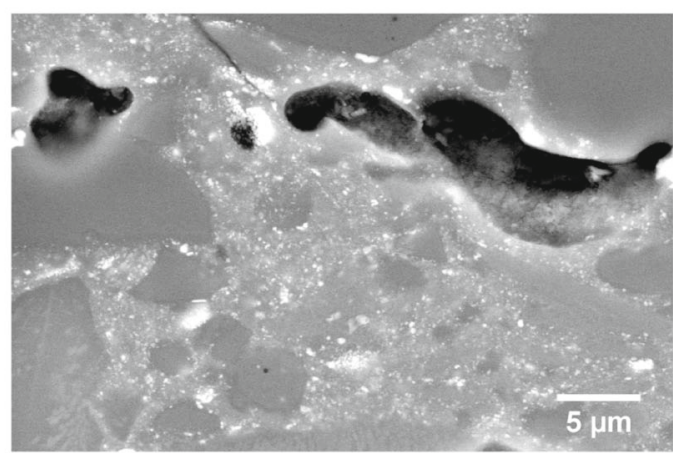

Fig. 14 Backscattered scanning electron micrographs at higher magnifications, ranging from $\times 10307$ to $\times 20343$, to depict details of the fired clay: (a) from sample "clay A $880^{\circ} \mathrm{C} x$ ", (b) from sample "clay B $880^{\circ} \mathrm{C} x$ ", (c) from sample "clay A $1100{ }^{\circ} \mathrm{C} x$ ", (d) from sample "clay B $1100^{\circ} \mathrm{C} x$ ”

Simultaneous vanishing of the muscovite phase is probably due to dehydroxylation and weakening the long range structural organization of the latter, as reported by Gridi-Bennadji et al. [53] and Barlow and Manning [54].

\subsection{Morphological analysis}

From a morphological viewpoint, the overall emerging picture is the following: at $880^{\circ} \mathrm{C}$ firing temperature, a contiguous matrix contains slit-type pores, quartz and feldspar grains, as well as platy muscovite inclusions; in clay A, also grains of decarbonated dolomite appear, and with a burning temperature of $1100{ }^{\circ} \mathrm{C}$ degenerate to pore coatings, see Figs. 12a, b and $13 \mathrm{a}, \mathrm{b}$. At $1100^{\circ} \mathrm{C}$ firing temperature, the pores become more ellipsoidal in shape. Regarding these SEM images, the following can be stated: the induced secondary porosity in clay A due to the decarbonation of dolomite and calcite (see [42, 49]), can be observed, while porosity in clay $\mathrm{B}$ at lower burning temperatures seems to be mainly driven by shrinkage of the clay matrix (Fig. 13). With higher burning temperatures, the formation of a melt phase leads to more ellipsoidal and spherical pores, compared to the sharp edged pores at a burning temperature of $880^{\circ} \mathrm{C}$, which is in accordance with observations by Cultrone et al. [47].

Quantitative morphological information for all distinguished material phases is given in Tables 7 and 8 , and reveals the preferential orientation to be with the extrusion axis, something which has already been observed by previous authors [7, 13]. In general, it can be stated that the more elongated the inclusions are, the more their preferential orientation is with the extrusion axis. While the less elongated inclusions (quartz, feldspar and decarbonized dolomite) show a huge weighted standard deviation of the preferential 
Table 7 Morphological information derived from SEM-EDX measurements: mean values and standard deviations of aspect ratios $a_{1} / \overline{a_{3}}$ and weighted standard deviations $\sigma_{\theta}$ of the orientation angles $\theta$ of the identified material phases for the four investigated fired clay samples

\begin{tabular}{|c|c|c|c|c|c|}
\hline & & \multicolumn{2}{|l|}{ Clay A } & \multicolumn{2}{|l|}{ Clay B } \\
\hline & & $880{ }^{\circ} \mathrm{C}$ & $1100{ }^{\circ} \mathrm{C}$ & $880{ }^{\circ} \mathrm{C}$ & $1100{ }^{\circ} \mathrm{C}$ \\
\hline \multirow[t]{2}{*}{ Quartz } & $a_{1} / \overline{a_{3}}$ & $1.722( \pm 0.161)$ & $1.867( \pm 0.055)$ & $1.769( \pm 0.260)$ & $1.783( \pm 0.031)$ \\
\hline & $\sigma_{\theta}$ & $35.71^{\circ}$ & $37.80^{\circ}$ & $42.26^{\circ}$ & $36.38^{\circ}$ \\
\hline \multirow[t]{2}{*}{ Feldspar } & $a_{1} / \overline{a_{3}}$ & $2.199( \pm 0.390)$ & $2.786( \pm 0.014)$ & $1.726( \pm 0.152)$ & $2.816( \pm 0.315)$ \\
\hline & $\sigma_{\theta}$ & $26.98^{\circ}$ & $30.58^{\circ}$ & $39.13^{\circ}$ & $37.37^{\circ}$ \\
\hline \multirow[t]{2}{*}{ Pores } & $a_{1} / \overline{a_{3}}$ & $2.668( \pm 0.452)$ & $2.350( \pm 0.383)$ & $3.881( \pm 0.344)$ & $3.671( \pm 0.373)$ \\
\hline & $\sigma_{\theta}$ & $31.02^{\circ}$ & $35.84^{\circ}$ & $39.08^{\circ}$ & $38.23^{\circ}$ \\
\hline \multirow[t]{2}{*}{ Dec. dolomite } & $a_{1} / \overline{a_{3}}$ & $1.664( \pm 0.149)$ & - & - & - \\
\hline & $\sigma_{\theta}$ & $29.80^{\circ}$ & - & - & - \\
\hline \multirow[t]{2}{*}{ Phyllosilica } & $a_{1} / \overline{a_{3}}$ & $6.414( \pm 1.508)$ & - & - & - \\
\hline & $\sigma_{\theta}$ & $23.96^{\circ}$ & - & - & - \\
\hline \multirow[t]{2}{*}{ Fe-Mg-phyllosilica } & $a_{1} / \overline{a_{3}}$ & $7.137( \pm 1.887)$ & $7.771( \pm 0.152)$ & $5.078( \pm 0.819)$ & - \\
\hline & $\sigma_{\theta}$ & $16.58^{\circ}$ & $26.57^{\circ}$ & $34.91^{\circ}$ & - \\
\hline
\end{tabular}

Table 8 Aspect ratios $a_{1} / a_{3}$ of the spheroids, back-calculated from the ellipses obtained from 2D images, using the approach outlined in Sect. 2.1

\begin{tabular}{|c|c|c|c|c|}
\hline & \multicolumn{2}{|l|}{ Clay A } & \multicolumn{2}{|l|}{ Clay B } \\
\hline & $880^{\circ} \mathrm{C}$ & $1100{ }^{\circ} \mathrm{C}$ & $880{ }^{\circ} \mathrm{C}$ & $1100{ }^{\circ} \mathrm{C}$ \\
\hline Quartz & 3.0850 & 2.6583 & 4.0084 & 2.5244 \\
\hline Feldspar & 3.4044 & 3.8083 & 3.1375 & 4.0701 \\
\hline Pores & 4.8245 & 3.7390 & 6.0695 & 5.7293 \\
\hline Dec. dolomite & 2.4598 & - & - & - \\
\hline Phyllosilica & 9.8605 & - & - & - \\
\hline Fe-Mg-phyllosilica & 10.8331 & 10.171 & 9.2121 & - \\
\hline
\end{tabular}

orientation, the elongated inclusions (phyllosilica) are aligned almost parallel to the extrusion axis.

\section{Conclusions}

Conclusively, our new phase identification protocol, stemming from the combination of SEM-EDX images with a preliminary conducted PXRD, is fully consistent with many previous investigations, while marking, at the same time, a new level of completeness, as the entire space of SEM-resolved microstructure is clearly assigned to one of up to seven phases with different shapes. Additionally, the chemical composition of the matrix phase has been calculated using obtained data by
PXRD and XRF measurements, confirming the assumption of finely dispersed quartz and feldspar particles below the resolution threshold of the SEM. Plus, these results are in line with the mineralogical analysis of the unfired raw material and the fired clay at different burning temperatures. Finally, the morphological evaluation of all material phases allows for a thorough description of the morphometrics of the latter. This opens the way towards a more quantitative understanding of structure-property relations in fired clay, in particular for mechanical and thermal properties predicted with tools of continuum micromechanics or homogenization theory [15]. The latter proved as very suitable for this purpose, in the context of various construction materials. With the presented identification of the material phases and their morphology, the first step towards such a physically based multiscale material model able to estimate macroscopic elastic and thermal properties of fired clay [26] is taken.

Acknowledgements Open access funding provided by TU Wien (TUW). The authors are thankful for the support by the "Klima- und Energiefonds" through the programme "Energie der Zukunft" and Österreichische Forschungsförderungsgesellschaft (FFG, Project Number 843897).

\section{Complianced with ethical standards}

Conflict of interest The authors declare that they have no conflict of interest. 
Open Access This article is licensed under a Creative Commons Attribution 4.0 International License, which permits use, sharing, adaptation, distribution and reproduction in any medium or format, as long as you give appropriate credit to the original author(s) and the source, provide a link to the Creative Commons licence, and indicate if changes were made. The images or other third party material in this article are included in the article's Creative Commons licence, unless indicated otherwise in a credit line to the material. If material is not included in the article's Creative Commons licence and your intended use is not permitted by statutory regulation or exceeds the permitted use, you will need to obtain permission directly from the copyright holder. To view a copy of this licence, visit http://creativecommons.org/licenses/by/4.0/.

\section{References}

1. Bergaya F, Lagaly G (2006) Chapter 1 general introduction: clays, clay minerals, and clay science. In: Faïza Bergaya BKT, Lagaly G (eds) Handbook of clay science, developments in clay science, vol 1, Elsevier, pp 1-18. https://doi. org/10.1016/S1572-4352(05)01001-9, http://www. sciencedirect.com/science/article/pii/S1572435205010019

2. Bergaya F, Lagaly G, Vayer M (2006) Chapter 12.10: cation and anion exchange. In: Faïza Bergaya BKT, Lagaly G (eds) Handbook of clay science, developments in clay science, vol 1, Elsevier, pp 979-1001. https://doi.org/10.1016/ S1572-4352(05)01036-6, http://www.sciencedirect.com/ science/article/pii/S1572435205010366

3. Pusch R (2006) Chapter 6 mechanical properties of clays and clay minerals. In: Faïza Bergaya BKT, Lagaly G (eds) Handbook of clay science, developments in clay science, vol 1, Elsevier, pp 247-260. https://doi.org/10.1016/S15724352(05)01006-8, http://www.sciencedirect.com/science/ article/pii/S1572435205010068

4. Coletti C, Cultrone G, Maritan L, Mazzoli C (2016) How to face the new industrial challenge of compatible, sustainable brick production: study of various types of commercially available bricks. Appl Clay Sci 124(Supplement C):219-226. https://doi.org/10.1016/j.clay.2016.02.014, http://www.sciencedirect.com/science/article/pii/ S0169131716300692

5. Jordán M, Montero M, Meseguer S, Sanfeliu T (2008) Influence of firing temperature and mineralogical composition on bending strength and porosity of ceramic tile bodies. Appl Clay Sci 42(1):266-271. https://doi.org/10. 1016/j.clay.2008.01.005, http://www.sciencedirect.com/ science/article/pii/S0169131708000148

6. Bennour A, Mahmoudi S, Srasra E, Boussen S, Htira N (2015) Composition, firing behavior and ceramic properties of the Sejnène clays (Northwest Tunisia). Appl Clay Sci 115:30-38. https://doi.org/10.1016/j.clay.2015.07.025, http://www.sciencedirect.com/science/article/pii/ S016913171530048X

7. Bourret J, Tessier-Doyen N, Guinebretiere R, Joussein E, Smith D (2015) Anisotropy of thermal conductivity and elastic properties of extruded clay-based materials: evolution with thermal treatment. Appl Clay Sci 116(Supplement C):150-157. https://doi.org/10.1016/j.clay.2015. 08.006, http://www.sciencedirect.com/science/article/pii/ S0169131715300739

8. Środń J (2006) Chapter 12.2 identification and quantitative analysis of clay minerals. In: Faïza Bergaya BKT, Lagaly G (eds) Handbook of clay science, developments in clay science, vol 1, Elsevier, pp 765-787. https://doi.org/10.1016/ S1572-4352(05)01028-7, http://www.sciencedirect.com/ science/article/pii/S1572435205010287

9. Wilson W, Sorelli L, Tagnit-Hamou A (2018a) Automated coupling of nanoindentation and quantitative energy-dispersive spectroscopy (ni-qeds): a comprehensive method to disclose the micro-chemo-mechanical properties of cement pastes. Cem Concre Res 103:49-65. https://doi.org/10. 1016/j.cemconres.2017.08.016, http://www.sciencedirect. com/science/article/pii/S0008884617305549

10. Wilson W, Sorelli L, Tagnit-Hamou A (2018b) Unveiling micro-chemo-mechanical properties of c-(a)-s-h and other phases in blended-cement pastes. Cem Concr Res 107:317-336. https://doi.org/10.1016/j.cemconres.2018.02. 010, http://www.sciencedirect.com/science/article/pii/ S000888461731089X

11. Hisseine OA, Wilson W, Sorelli L, Tolnai B, Tagnit-Hamou A (2019) Nanocellulose for improved concrete performance: a macro-to-micro investigation for disclosing the effects of cellulose filaments on strength of cement systems. Constr Build Mater 206:84-96. https://doi.org/10.1016/j. conbuildmat.2019.02.042, http://www.sciencedirect.com/ science/article/pii/S095006181930337X

12. Chen JJ, Sorelli L, Vandamme M, Ulm FJ, Chanvillard G (2010) A coupled nanoindentation/sem-eds study on low water/cement ratio portland cement paste: evidence for $\mathrm{c}-\mathrm{s}-$ $\mathrm{h} / \mathrm{ca}(\mathrm{oh}) 2$ nanocomposites. J Am Ceram Soc 93(5):1484-1493. https://doi.org/10.1111/j.1551-2916. 2009.03599.x, https://ceramics.onlinelibrary.wiley.com/ doi/abs/10.1111/j.1551-2916.2009.03599.x, https:// ceramics.onlinelibrary.wiley.com/doi/pdf/10.1111/j.15512916.2009.03599.x

13. Krakowiak KJ, Lourenço PB, Ulm FJ (2011) Multitechnique investigation of extruded clay brick microstructure. J Am Ceram Soc 94(9):3012-3022. https://doi.org/10.1111/ j.1551-2916.2011.04484.x

14. Hill R (1965) Continuum micro-mechanics of elastoplastic polycrystals. J Mech Phys Solids 13(2):89-101. https://doi. org/10.1016/0022-5096(65)90023-2, http://www. sciencedirect.com/science/article/pii/0022509665900232

15. Zaoui A (2002) Continuum micromechanics: survey. J Eng Mech (ASCE) 128(8):808-816

16. Giraud A, Gruescu C, Do D, Homand F, Kondo D (2007) Effective thermal conductivity of transversely isotropic media with arbitrary oriented ellipsoidal inhomogeneities. Int J Solids Struct 44(9):2627-2647. https://doi.org/10. 1016/j.ijsolstr.2006.08.011

17. Constantinides G, Ulm FJ (2004) The effect of two types of $\mathrm{C}-\mathrm{S}-\mathrm{H}$ on the elasticity of cement-based materials: results from nanoindentation and micromechanical modeling. Cem Concr Res 34(1):67-80. https://doi.org/10.1016/S00088846(03)00230-8, http://www.sciencedirect.com/science/ article/pii/S0008884603002308 
18. Pichler B, Hellmich C (2011) Upscaling quasi-brittle strength of cement paste and mortar: a multi-scale engineering mechanics model. Cem Concr Res 41(5):467-476. https://doi.org/10.1016/j.cemconres.2011.01.010, http:// www.sciencedirect.com/science/article/pii/ S0008884611000111

19. Hofstetter K, Hellmich C, Eberhardsteiner J (2005) Development and experimental validation of a continuum micromechanics model for the elasticity of wood. Eur J Mech A/Solids 24(6):1030-1053. https://doi.org/10.1016/j. euromechsol.2005.05.006, http://www.sciencedirect.com/ science/article/pii/S0997753805000963

20. Bader T, Hofstetter K, Hellmich C, Eberhardsteiner J (2010) Poromechanical scale transitions of failure stresses in wood: from the lignin to the spruce level. ZAMM-J Appl Math Mech / Zeitschrift für Angewandte Mathematik und Mechanik 90(10-11):750 - 767. https://doi.org/10.1002/ zamm.201000045, https://onlinelibrary.wiley.com/doi/abs/ 10.1002/zamm.201000045, https://onlinelibrary.wiley. com/doi/pdf/10.1002/zamm.201000045

21. Fritsch A, Hellmich C (2007) "Universal" microstructural patterns in cortical and trabecular, extracellular and extravascular bone materials: micromechanics-based prediction of anisotropic elasticity. J Theor Biol 244(4):597620. https://doi.org/10.1016/j.jtbi.2006.09.013, http://www. sciencedirect.com/science/article/pii/S0022519306004206

22. Fritsch A, Dormieux L, Hellmich C, Sanahuja J (2009) Mechanical behavior of hydroxyapatite biomaterials: an experimentally validated micromechanical model for elasticity and strength. J Biomed Mater Res Part A 88A(1):149-161. https://doi.org/10.1002/jbm.a.31727, https://onlinelibrary.wiley.com/doi/abs/10.1002/jbm.a.

31727, https://onlinelibrary.wiley.com/doi/pdf/10.1002/ jbm.a.31727

23. Abdalrahman T, Scheiner S, Hellmich C (2015) Is trabecular bone permeability governed by molecular orderinginduced fluid viscosity gain? Arguments from re-evaluation of experimental data in the framework of homogenization theory. J Theor Biol 365:433-444. https://doi.org/10.1016/j. jtbi.2014.10.011, http://www.sciencedirect.com/science/ article/pii/S0022519314006006

24. Schultz L (1964) Quantitative interpretation of mineralogical composition from X-ray and chemical data for the Pierre Shale. Tech. rep., USGS Publications Warehouse. https://doi.org/10.3133/pp391C, 391C

25. Kariem H, Füssl J, Kiefer T, Jäger A, Hellmich C (2020) The viscoelastic behaviour of material phases in fired clay identified by means of grid nanoindentation. Constr Build Mater 231:117066. https://doi.org/10.1016/j.conbuildmat.2019. 117066, http://www.sciencedirect.com/science/article/pii/ S0950061819325085

26. Kiefer T, Füssl J, Kariem H, Konnerth J, Gaggl W, Hellmich C (2020) A multi-scale material model for the estimation of the transversely isotropic thermal conductivity tensor of fired clay bricks. J Eur Ceram Soc. https://doi.org/10.1016/j. jeurceramsoc.2020.05.018, http://www.sciencedirect.com/ science/article/pii/S0955221920303691

27. Oliver W, Pharr G (1992) An improved technique for determining hardness and elastic modulus using load and displacement sensing indentation experiments. J Mater Res 7(6):1564-1583. https://doi.org/10.1557/JMR.1992.1564
28. Fischer H (2005) Quantitative determination of heat conductivities by scanning thermal microscopy. Thermochim Acta 425(1-2):69-74. https://doi.org/10.1016/j.tca.2004. 06.005

29. Vander Voort GF (2007) BUEHLER ${ }^{\circledR}$ Sum-Met ${ }^{\text {TM }}$ - the science behind materials preparation. Buehler Ltd

30. Rietveld HM (1969) A profile refinement method for nuclear and magnetic structures. J Appl Crystallogr 2:65-71

31. Madsen IC, Scarlett NVY, Kern A (2011) Description and survey of methodologies for the determination of amorphous content via X-ray powder diffraction. Zeitschrift für Kristallographie-Crystalline Materials 226(12):944-955. https://doi.org/10.1524/zkri.2011.1437

32. Halliday D, Resnick R, Walker J (2010) Fundamentals of physics. Wiley

33. Russ JC (1984) Fundamentals of energy dispersive X-ray analysis. Butterworth-Heinemann

34. Friel JJ, Lyman CE (2006) Tutorial review: X-ray mapping in electron-beam instruments. Microsc Microanal 12(1):2-25. https://doi.org/10.1017/S1431927606060211

35. Newbury DE, Ritchie NWM (2013) Is scanning electron microscopy/energy dispersive X-ray spectrometry (SEM/ EDS) quantitative? Scanning 35(3):141-168. https://doi. org/10.1002/sca.21041, https://onlinelibrary.wiley.com/ doi/abs/10.1002/sca.21041, https://onlinelibrary.wiley. com/doi/pdf/10.1002/sca.21041

36. Goldstein J, Newbury DE, Joy DC, Lyman CE, Echlin P, Lifshin E, Sawyer L, Michael J (2003) Scanning electron microscopy and X-ray microanalysis, 3rd edn. Springer US. https://doi.org/10.1007/978-1-4615-0215-9

37. Otsu N (1979) A threshold selection method from gray-level histograms. IEEE Trans Syst Man Cybern 9(1):62-66. https://doi.org/10.1109/TSMC.1979.4310076

38. Schneider CA, Rasband WS, Eliceiri KW (2012) NIH image to ImageJ: 25 years of image analysis. Nat Meth 9(7):671-675. https://doi.org/10.1038/nmeth.2089

39. Welton JE (1984) SEM petrology atlas. American Association of Petroleum Geologists. https://doi.org/10.1306/ Mth4442, http://geoscienceworld.org/content/ 9781629811611/9781629811611

40. Yen JC, Chang FJ, Chang S (1995) A new criterion for automatic multilevel thresholding. IEEE Trans Image Process 4(3):370-378. https://doi.org/10.1109/83.366472

41. Lafuente B, Downs RT, Yang H, Stone N (2015) Highlights in mineralogical crystallography, De Gruyter, chap The power of databases: the RRUFF project, pp 1-29. https:// doi.org/10.1515/9783110417104-003, https://www. amazon.com/Highlights-Mineralogical-CrystallographyThomas-Armbruster/dp/3110417049?SubscriptionId= AKIAIOBINVZYXZQZ2U3A\&tag=chimbori05$20 \&$ linkCode $=x m 2 \&$ camp $=2025 \&$ creative $=$ $165953 \&$ creativeASIN=3110417049

42. Riccardi M, Messiga B, Duminuco P (1999) An approach to the dynamics of clay firing. Appl Clay Sci 15(3):393-409. https://doi.org/10.1016/S0169-1317(99)00032-0, http:// www.sciencedirect.com/science/article/pii/ S0169131799000320

43. Nodari L, Marcuz E, Maritan L, Mazzoli C, Russo U (2007) Hematite nucleation and growth in the firing of carbonaterich clay for pottery production. J Eur Ceram Soc 27(16):4665-4673. https://doi.org/10.1016/j.jeurceramsoc. 
2007.03.031, http://www.sciencedirect.com/science/article/ pii/S095522190700324X

44. Duminuco P, Messiga B, Riccardi M (1998) Firing process of natural clays. Some microtextures and related phase compositions. Thermochim Acta 321(1):185-190. https:// doi.org/10.1016/S0040-6031(98)00458-4, http://www. sciencedirect.com/science/article/pii/S0040603198004584

45. Fischer P (1987/1988) Formation of the ceramic body in heavy clay products in firing-Part 2: formation of the ceramic body structure. Ziegeleitechnisches Jahrbuch pp 96-108

46. Maggetti M (1982) Phase analysis and its significance for technology and origin. Archaeological ceramics, pp 121-133

47. Cultrone G, Rodriguez-Navarro C, Sebastian E, Cazalla O, De La Torre MJ (2001) Carbonate and silicate phase reactions during ceramic firing. Eur J Miner 13(3):621-634. https://doi.org/10.1127/0935-1221/2001/0013-0621

48. Peters T, Jenni J (1973) Mineralogische Untersuchungen über das Brennverhalten von Ziegeltonen. In: Beiträge zur Geologie der Schweiz, Schweizerische Geotechnische Kommission, Kümmerly \& Frey, Geographischer Verlag, Bern, vol 50

49. Cultrone G, Sebastián E, Elert K, de la Torre MJ, Cazalla O, Rodriguez-Navarro C (2004) Influence of mineralogy and firing temperature on the porosity of bricks. J Eur Ceram Soc 24(3):547-564. https://doi.org/10.1016/S09552219(03)00249-8, http://www.sciencedirect.com/science/ article/pii/S0955221903002498

50. Maritan L, Nodari L, Mazzoli C, Milano A, Russo U (2006) Influence of firing conditions on ceramic products: experimental study on clay rich in organic matter. Appl Clay Sci 31(1):1-15. https://doi.org/10.1016/j.clay.2005.08.007, http://www.sciencedirect.com/science/article/pii/ S0169131705001122

51. Zouaoui H, Lecomte-Nana GL, Krichen M, Bouaziz J (2017) Structure, microstructure and mechanical features of ceramic products of clay and non-plastic clay mixtures from Tunisia. Appl Clay Sci 135:112-118. https://doi.org/10. 1016/j.clay.2016.09.012, http://www.sciencedirect.com/ science/article/pii/S0169131716303817

52. Kariem H, Hellmich C, Kiefer T, Jäger A, Füssl J (2018) Micro-CT-based identification of double porosity in fired clay ceramics. J Mater Sci 53(13):9411-9428. https://doi. org/10.1007/s10853-018-2281-9

53. Gridi-Bennadji F, Beneu B, Laval J, Blanchart P (2008) Structural transformations of muscovite at high temperature by X-ray and neutron diffraction. Appl Clay Sci 38(3):259-267. https://doi.org/10.1016/j.clay.2007.03.003, http://www.sciencedirect.com/science/article/pii/ S016913170700066X

54. Barlow S, Manning D (1999) Influence of time and temperature on reactions and transformations of muscovite mica. Br Ceram Trans 98(3):122-126. https://doi.org/10. $1179 / 096797899680327$

Publisher's Note Springer Nature remains neutral with regard to jurisdictional claims in published maps and institutional affiliations. 